\title{
Transient periodic behaviour related to a saddle-node bifurcation
}

\author{
Ruud van Damme and Theo P Valkering \\ Centre for Theoretical Physics, University of Twente, PO Box 217, 7500 AE Enschede, \\ The Netherlands
}

Received 19 December 1986, in final form 23 February 1987

\begin{abstract}
We investigate transient periodic orbits of dissipative invertible maps of $\mathbb{R}^{2}$. Such orbits exist just before, in parameter space, a saddle-node pair is formed. We obtain numerically and analytically simple scaling laws for the duration of the transient, and for the region of initial conditions which evolve into transient periodic orbits. An estimate of this region is then obtained by the construction-after extension of the map to $\mathbb{C}^{2}$-of the stable manifolds of the two complex saddles in $\mathbb{C}^{2}$ that bifurcate into the real saddle-node pair.
\end{abstract}

\section{Introduction}

An orbit in a dissipative system can show transient periodic behaviour, i.e. before the orbit falls down onto the final attractor it seems periodic for a long, but finite, time.

Saddle-node bifurcations of periodic orbits are common for dynamical dissipative systems. Here we demonstrate for maps of the real plane that each saddle-node bifurcation is preceded by this transient periodic behaviour. Essential for our description of this phenomenon is that in such a bifurcation process two complex conjugate periodic orbits in $\mathbb{C}^{2}$ move, as a function of a control parameter, towards the real plane; they coincide and then separate in $\mathbb{R}^{2}$ as a saddle and a node (Guckenheimer and Holmes 1983). Long periodic transients then occur just before the saddle-node bifurcation takes place, when the two complex periodic orbits are close enough to the real plane to influence the orbit in $\mathbb{R}^{2}$.

It turns out that, for parameter values close to this bifurcation point, the region of initial points in phase space that exhibit this transient periodic behaviour can be estimated by the construction of the boundary of the inset of the fixed point at the bifurcation point. As an alternative we make such an estimate by the construction of the stable manifold of one of the two saddle periodic orbits in $\mathbb{C}^{2}$. Roughly speaking the projections on $\mathbb{R}^{2}$ of points on this manifold, whose distance to $\mathbb{R}^{2}$ is minimal, form the boundary of the transient periodic region.

Similarly to the length of intermittency in one-dimensional maps (Schuster 1984), the length of transient periodic behaviour scales with the inverse of the root of the distance of the complex saddles to the real plane (cq line). The width of the boundary of the transient periodic region scales with this same root. 
We investigate this phenomenon for the invertible real quadratic map, $H$, with constant Jacobian, $b$ with $0<|b|<1$,

$$
\left.\begin{array}{l}
x^{\prime}=(1+b) x+2 x^{2}-b y+\beta \\
y^{\prime}=x
\end{array}\right\} \equiv H\{x, y\}
$$

which describes a typical saddle-node bifurcation if $\beta$ approaches zero from above. The map has two complex fixed points if $\beta>0$, at a distance proportional to $\sqrt{ } \beta$ from the real plane. The saddle-node bifurcation occurs at the origin if $\beta=0$.

\section{Asymptotics of the trajectories before the saddle-node bifurcation}

Below, the following results are proved for the map (1). If $\beta>0$ every orbit diverges. If $\beta=0$ the plane is divided in two parts: the inset $B_{0}$ of the elliptic fixed point at the origin, and its complement $B_{\infty}$, in which every orbit diverges. $B_{0}$ is a closed simply connected set. Its boundary is an analytic curve that coincides with the set of points which converge to the origin along its stable direction. As a result $B_{0}$ can be obtained by construction of this boundary (cf the appendix).

Equations $(1 a, b)$ yield for a trajectory $\left\{x_{n}, y_{n}\right\}$

$$
x_{n+1}-b y_{n+1}=x_{n}-b y_{n}+2 x_{n}^{2}+\beta \geqslant x_{n}-b y_{n}+\beta \text {. }
$$

Therefore $x_{n}-b y_{n}$ increases, at least with a constant amount $\beta$ if $\beta>0$. Hence each orbit diverges in this case. If $\beta=0, x_{n}-b y_{n}$ either converges, or tends to infinity. In the first case, if $n \rightarrow \infty$, then $x_{n}^{2} \rightarrow 0$ and consequently both $x_{n}$ and $y_{n}\left(=x_{n-1}\right)$ converge to zero. Thus if $\beta=0$ an orbit either goes to the origin, or diverges. Furthermore observe that in the latter case each orbit, after sufficiently many iterations, arrives in the open set $(\{x, y\} \mid x-b y>0)$. Thus $B_{\infty}$ is open and consequently $B_{0}$ is closed.

The manifold $W_{0}^{s}$ is defined as the set of points in $B_{0}$ whose orbits approach the fixed point along the stable direction. $W_{0}^{s}$ coincides with the boundary of $B_{0}$. Consider
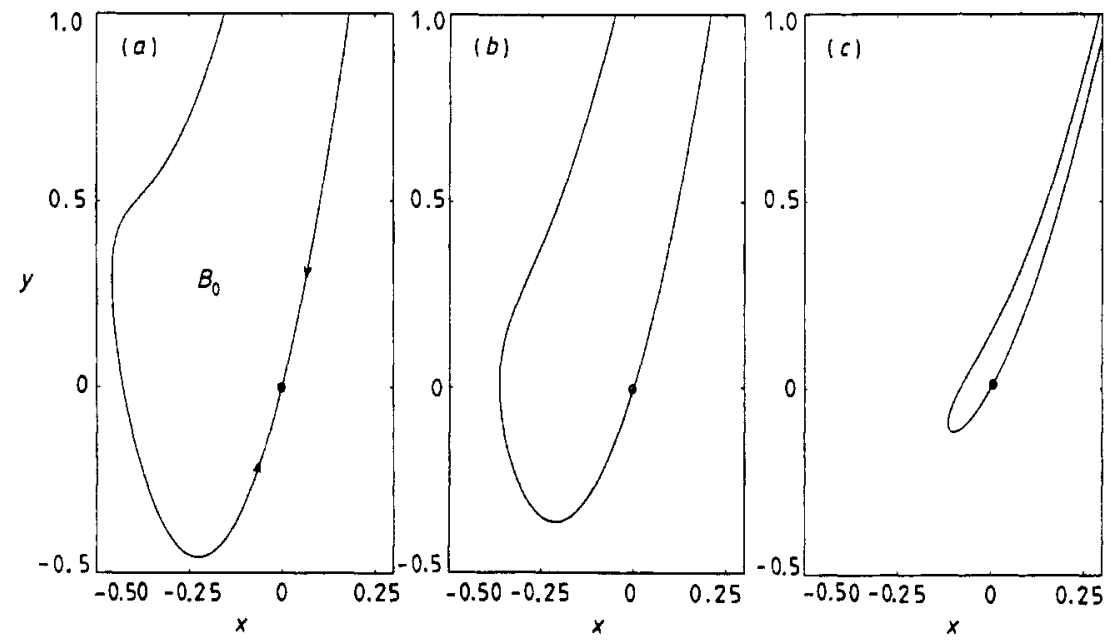

Figure 1. Phase diagram at the saddle-node bifurcation. The boundary $W_{0}^{s}$ of $B_{0}$ of map $H$ for different values of the Jacobian $b:(a) b=0.25$, (b) $b=0.3$, (c) $b=0.55$. The saddle-node is depicted as $\bigcirc$. Observe that $B_{0}$ is smaller for increasing $b$. 

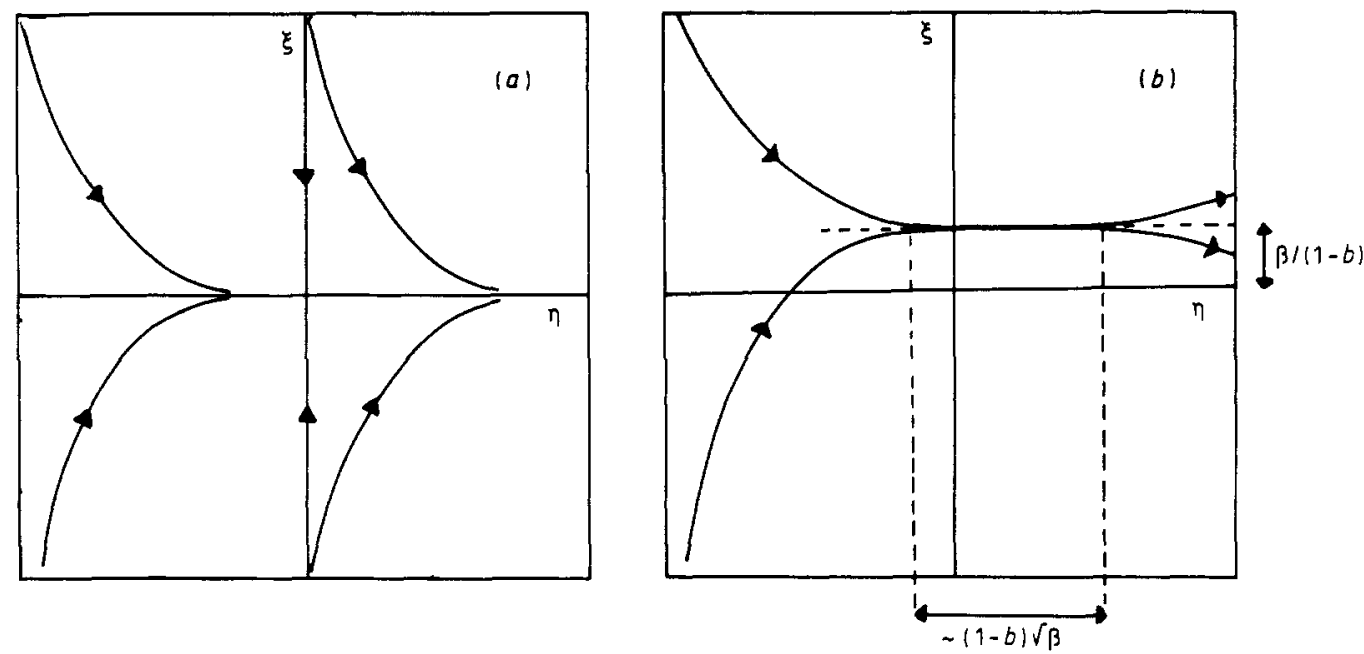

Figure 2. (a) Behaviour of orbits near the origin of map $H$ for $\beta=0$ as found from (3). (b) Behaviour of orbits near the origin of map $H$ for $\beta>0$. The drawn curves smoothly connect elements of an orbit.

$z_{0} \in \partial B_{0}$. Then the local behaviour (cf figure 2) of the orbit of $H$ near the fixed point guarantees that $z_{0} \in W_{0}^{s}$. Conversely $W_{0}^{s} \subset B_{0}$ by definition. In the appendix a construction method for $W_{0}^{\text {s }}$ is given.

For small $\beta$, the local behaviour of orbits in the neighbourhood of the origin is determined by (see figure 2 )

$$
\begin{aligned}
& \eta^{\prime}=\eta+2 \eta^{2} /(1-b)^{2}+\beta+\operatorname{Hot}(\xi, \eta) \\
& \xi^{\prime}=b \xi-4 b \eta \xi /(1-b)^{2}+\beta+\operatorname{HOT}(\xi, \eta)
\end{aligned}
$$

which relations are obtained from $(1 a)$ by a suitable coordinate transformation of the form

$$
\begin{aligned}
& \xi=x-b y+\text { HOT } \\
& \eta=x-y+\text { HOT. }
\end{aligned}
$$

Observe that for $\beta=0$ the first two terms in the RHS of $(3 a)$ and $(3 b)$ are the normal form of (1) (Arnold 1977) which is non-linear since the linearised map has an eigenvalue equal to one.

\section{Transient periodic behaviour: numerical observations}

If $\beta>0$ every orbit goes to infinity. However, there is a large region of initial points whose orbits are trapped for a long time in the neighbourhood of the origin. Here we define, and calculate for various $b$ and $\beta$, the trapping time for each orbit. If $\beta \ll 1$ the area with long trapping time appears to be well approximated by $B_{0}$, constructed at $\beta=0$. The long trapping timescales with $1 / \sqrt{ } \beta$. The width (for transversal intersection) of the transition region between initial points with long and short trapping time is proportional to $\sqrt{ } \beta$. 
The trapping time of a given initial condition is defined as the time to reach the region $A$ where the map is essentially quadratic: $x^{\prime} \approx 2 x^{2}, y^{\prime}=x$

$$
A \equiv\left(\{x, y\} \mid x-b y>0, x>R_{0}\right)
$$

where $R_{0}$ is a sufficiently large constant. If defined in this way the trapping time is a discontinuous function of the initial condition. To define a continuous trapping time, observe that the approximate map $x_{n+1}=2 x_{n}^{2}$ in $A$ is exactly solvable, $x_{n}=$ $\frac{1}{2} \exp \left[2^{n} \ln \left(2 x_{0}\right)\right]$. Now define for a given initial position the trapping time $\tau$ by

$$
\tau\left(\left\{x_{0}, y_{0}\right\}\right)=N+t
$$

where $N$ is such that $\left\{x_{N+1}, y_{N+1}\right\} \in A,\left\{x_{N}, y_{N}\right\} \notin A$ and $t$ is given by

$$
R_{0}=\frac{1}{2} \exp \left[2^{t} \ln \left(2 x_{N}\right)\right] \text {. }
$$

Although $\tau$ is still not continuous, this definition satisfies for numerical purposes. Figure 3 shows typical examples of $\tau\left(\left\{x_{0}, y_{0}\right\}\right)$ obtained in this way, for increasing $\beta$ and fixed $b=0.3$.
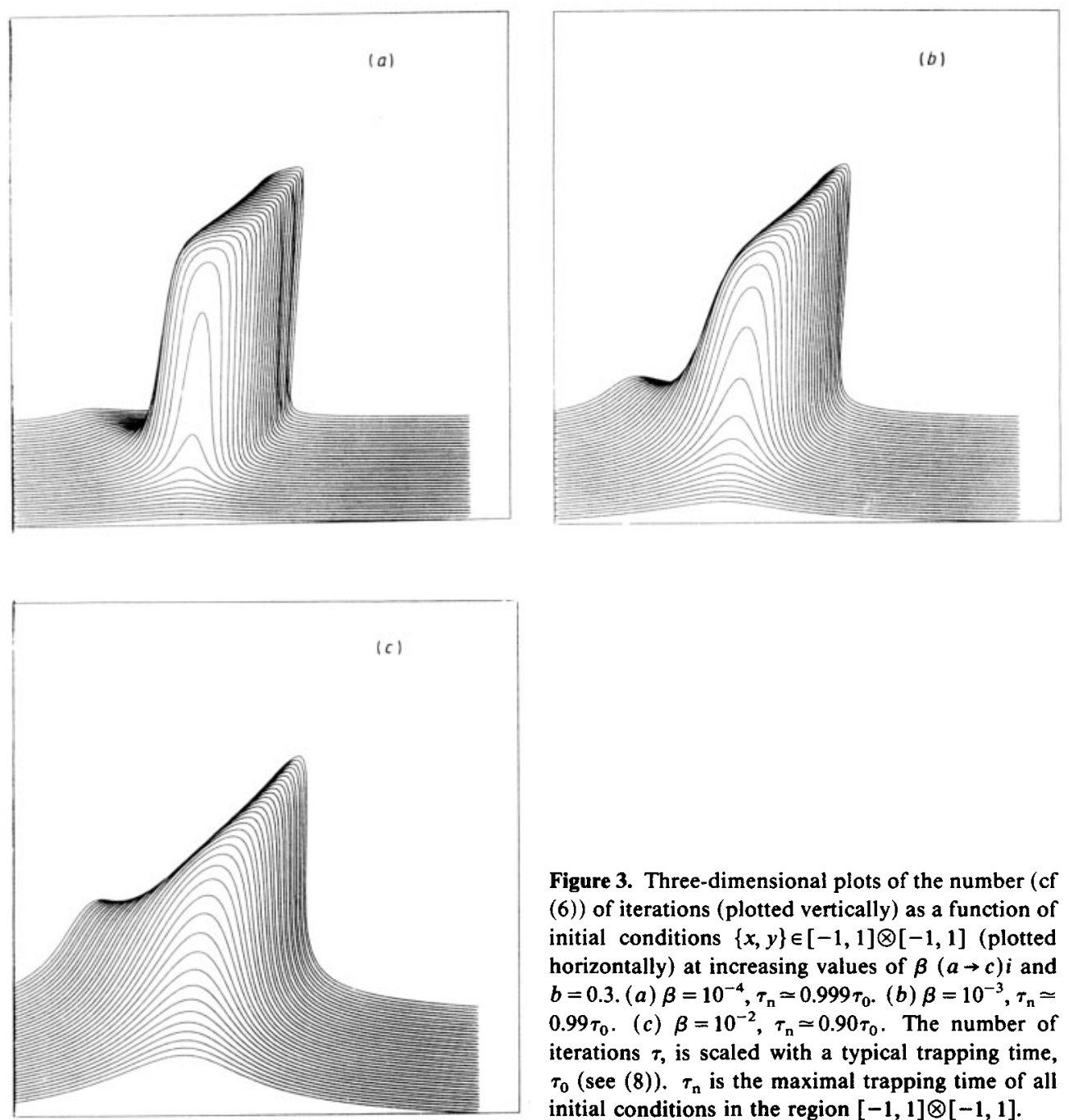

Figure 3. Three-dimensional plots of the number (cf (6)) of iterations (plotted vertically) as a function of initial conditions $\{x, y\} \in[-1,1] \otimes[-1,1]$ (plotted horizontally) at increasing values of $\beta(a \rightarrow c) i$ and $b=0.3$. (a) $\beta=10^{-4}, \tau_{\mathrm{n}} \simeq 0.999 \tau_{0}$. (b) $\beta=10^{-3}, \tau_{\mathrm{n}} \simeq$ $0.99 \tau_{0}$. (c) $\beta=10^{-2}, \tau_{n} \simeq 0.90 \tau_{0}$. The number of iterations $\tau$, is scaled with a typical trapping time, $\tau_{0}($ see $(8)), \tau_{n}$ is the maximal trapping time of all initial conditions in the region $[-1,1] \otimes[-1,1]$. 
Observe that in particular for small $\beta$ there exists a rather pronounced plateau of points with long trapping time. If $\beta$ increases the slopes become less steep and the maximal value of $\tau$ (in units of the typical trapping time $\tau_{0}$ (see (8)) diminishes. Furthermore a second ridge, which exists for all $\beta>0$, becomes relatively more important.

We obtained the following results from our numerical computations varying $b$ and letting $\beta$ approach zero from above.

(i) Maximal trapping time $\tau_{0}$ scales as

$$
\tau_{0} \sim(1-b) / \sqrt{ } \beta \text {. }
$$

The constant of proportionality in (8), of course, depends on the chosen range of initial conditions and on the choice of $R_{0}$. However it is very insensitive for both choices and is numerically very close to $\pi / \sqrt{ } 2$, which is confirmed by the analytical analysis below. In the following we define $\tau_{0}=\pi(1-b) / \sqrt{ }(2 \beta)$.

(ii) The central line $C$ of the transition region, i.e. the contour line with $\tau=\tau_{0} / 2$, coincides to a good approximation with $W_{0}^{\mathrm{s}}$, the boundary of the inset, if $\beta=0$. The error is of the order of $\beta$, and therefore small.

(iii) The slope $s$ of $\tau(\{x, y\})$ at $\mathrm{C}$ scales as

$$
s \sim 1 / \sqrt{ } \beta .
$$

Consequently the width of the transition is $O(\sqrt{ } \beta)$. As a result the shift, mentioned in result (ii) is negligible. Note that the proportionality constant in (9) varies along the central line $C$ (cf figure 4).

Finally we remark that the trajectory of each initial point which shows transient periodic behaviour reaches the neighbourhood of the origin in a few steps and stays there for most of the trapping time. Furthermore, 'relevant' transient periodic behaviour occurs in a small range of the parameter: $0<\beta \leqslant 10^{-2}$.

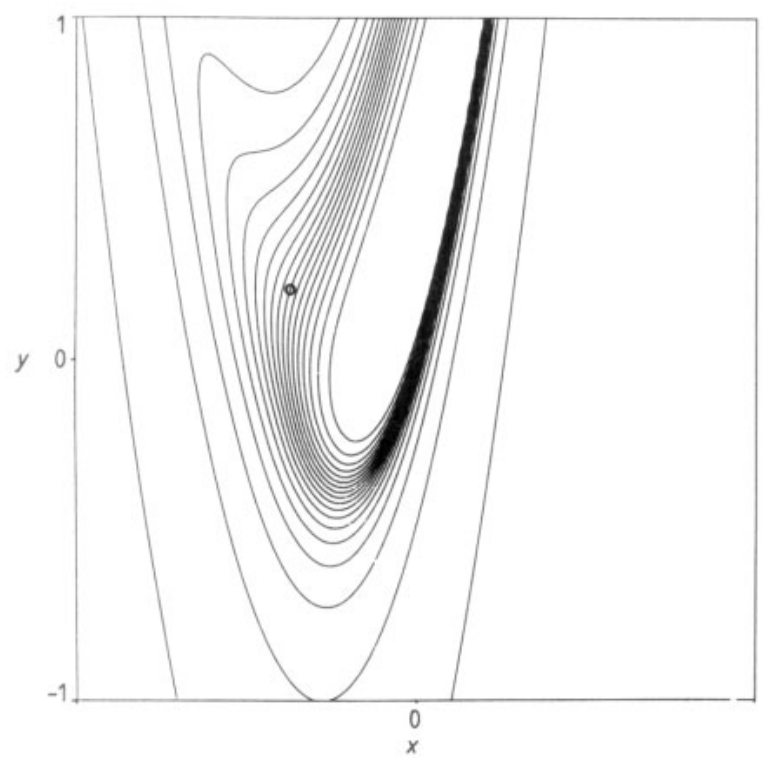

Figure 4. Contour lines of the 'landscape' in figure $3(b)$ for $b=0.3, \beta=10^{-3}$. The line with trapping time $\tau_{0} / 2, \mathrm{C}$, is indicated with $O$. 


\section{Analytic description of the observed results}

The scaling results are described quantitatively by the analytic solution of a map that approximates the trajectories in the neighbourhood of the origin. To find the transient periodic region for small values of $\beta$, we extend map (1) to a map from $\mathbb{C}^{2} \rightarrow \mathbb{C}^{2}$. We demonstrate that the complex stable manifold of one of the complex conjugate saddles before the bifurcation determines the region of transient periodic behaviour.

The local behaviour of the map $H$ at the origin is derived from $(3 a)$ and $(3 a)$. For a trajectory that passes through a small neighbourhood of the origin, $\xi_{n}$ tends to $\beta /(1-b)$ with $b^{n}$ and effectively map (1) reduces to the one-dimensional map $(3 a)$. The latter is approximated up to orders $\eta^{3}$ by

$$
\eta^{\prime}=\eta /(1-a \eta)+\beta
$$

where $a=2 /(1-b)^{2}$. This map is exactly solvable: consider the linear map of the plane

$$
\left(\begin{array}{c}
v^{\prime} \\
w^{\prime}
\end{array}\right)=\left(\begin{array}{cc}
1-a \beta & \beta \\
-a & 1
\end{array}\right)\left(\begin{array}{l}
v \\
w
\end{array}\right) \equiv U\left(\begin{array}{l}
v \\
w
\end{array}\right) .
$$

One readily verifies that there is a one-to-one correspondence between an orbit $\left(\eta_{0}, \eta_{1}, \ldots\right)$ of $(10)$ and the equivalence class of orbits $\left(\left\{v_{0}, w_{0}\right\},\left\{v_{1}, w_{1}\right\}, \ldots\right)$ of $(11)$, defined by $\eta_{0}=v_{0} / w_{0}$. Since (11) is linear, it can be solved. In this way we obtain for (10) the solution, $\left(\ldots, \eta_{n}, \ldots\right)$

$$
\eta_{n} \equiv \bar{\eta}(n)=\left(A \lambda^{n}+A^{*} \lambda^{* n}\right) /\left(B \lambda^{n}+B^{*} \lambda^{* n}\right) \text {. }
$$

Here $\lambda$ is an eigenvalue of $U$

$$
\lambda=\exp (\mathrm{i} \phi) \quad \phi=\cos ^{-1}(1-a \beta / 2) \approx \sqrt{ }(a \beta)
$$

and $A$ and $B$ are components of the eigenvector. Then $\left\{v_{0}, w_{0}\right\}=\left\{A+A^{*}, B+B^{*}\right\}$.

To interpret these results, recall that (10) approximates to (3a) if $\left|\eta^{3}\right|<\left|\eta^{2}\right|$ and $\left|\eta^{3}\right|<\beta$. Both inequalities are satisfied if $|\eta|<\sqrt{ } \beta<1$ (cf also figure 2). The time needed for an orbit $\left(\eta_{0}, \eta_{1}, \ldots\right)$ to go from $\eta_{0}$ to $\sqrt{ } \beta$ is $\tau\left(\eta_{0}\right)=\bar{\eta}(\sqrt{ } \beta)-\bar{\eta}\left(\eta_{0}\right), \bar{\eta}(\eta)$ being the inverse of $\bar{\eta}(n)$ in (12). Clearly from figure $5, \tau(-\sqrt{ } \beta)=\bar{n}(\sqrt{ } \beta)-\bar{n}(-\sqrt{ } \beta) \approx$ $\pi(1-b) / \sqrt{ }(2 \beta)$, which is in agreement with the value which was found numerically (cf (8)). Furthermore observe that $\bar{\eta}(n)$ also predicts the observed values for the shift proportional to $\beta$ and the slope $s$ (results (ii) and (iii) of $\S 3$ ).

To determine the trapping region, consider (1) as a map from $\mathbb{C}^{2} \rightarrow \mathbb{C}^{2}$. If $\beta>0$ this map has fixed points at

$$
x=y= \pm \mathrm{i} \sqrt{ }(\beta / 2)
$$

with eigenvalues determined by the equation

$$
\lambda+b / \lambda=1+b \pm 4 \mathrm{i} \sqrt{ }(\beta / 2) .
$$

Each fixed point is a saddle: one of the two eigenvalues (in absolute value) is larger than one and the other smaller than $b$. If $\beta$ tends to zero, these two points approach the real plane. At $\beta=0$ the two points merge into one real fixed point.

We now show that the stable manifold of one of the complex fixed points, $W^{\text {s }}$, can be used to predict the boundary of the region of transient periodic behaviour. For a (complex) saddle this stable manifold is the set $w \in \mathbb{C}^{2}$ such that $H^{n} w$ converges to the fixed point for $n \rightarrow \infty$. In the appendix we show the existence of an analytic transformation $z(u), \mathbb{C}^{1} \rightarrow \mathbb{C}^{2}$, whose range constitutes $\bar{W}^{s}$. Similarly, if $\beta=0$, a set $\bar{W}_{0}^{\mathrm{s}}$ is defined in $\mathbb{C}^{2}$. The intersection of this set with $\mathbb{R}^{2}$ is $W_{0}^{s}$ (cf figure 1 ). 


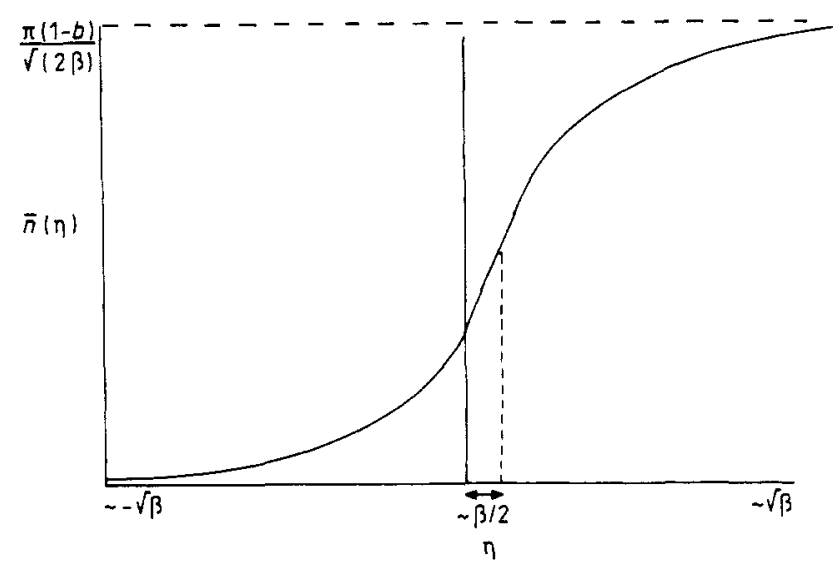

Figure 5. A schematic plot of $\bar{n}(\eta)$ for (10) calculated from (12) for some $b, \beta$ pair with very small $\beta, \beta \gg \beta$. Observe that the thickness and shift of the transition region agree with the numerical results $(8)$ and $(9)$.

To predict the boundary of the transient periodic region, recall that for a point $w \in \mathbb{C}^{2}, \operatorname{Re}(w)$ is its projection on $\mathbb{R}^{2}$ and $\|\operatorname{Im}(w)\|$ is its distance to $\mathbb{R}^{2}(\|\|$ is the Euclidean norm). Now consider for $\beta=0$ a line $l$ in $\mathbb{R}^{2}$ which crosses $W_{0}^{\text {s }}$ transversally. When we determine $w \in \bar{W}_{0}^{s}$ such that $\operatorname{Re}(w) \in l$, then the distance $\|\operatorname{Im}(w)\|$ to $l$ is minimal, i.e. zero, at the intersection of $l$ and $W_{0}^{s}$. Figure 6 shows $\|\operatorname{Im}(w)\|$ along a particular line $l$. Similarly, we expect for $\beta>0$, that when we cross the central line $C$ transversally along the curve $l$, the distance $\|\operatorname{Im}(w)\|$ with $w \in W^{s}$ and $\operatorname{Re}(w) \in l$, is minimal at the intersection point. Figure 7 confirms this claim. Observe that there are several branches. The branches for which the distance tends to zero if $\beta \downarrow 0$, correspond to the central line $C$. Positions on the other branches whose distance is minimal correspond to points in the real plane whose change in trapping time is maximal. The

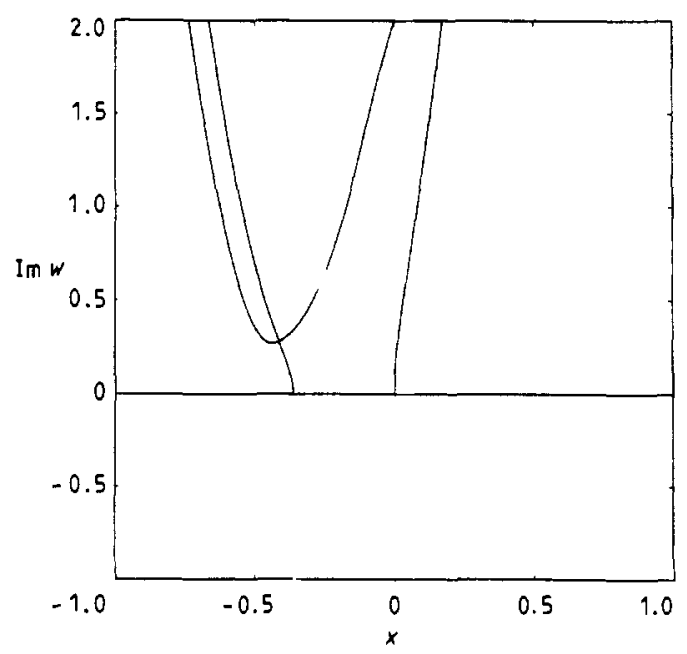

Figure 6. $\|\operatorname{lm}(w)\|$ plotted against the $x$ component of $\operatorname{Re}(w)$ for $\beta=0, y=0 ; w \in W^{s}$ and the projection of $w$ on $\mathbb{R}^{2}$ is in $l$ (cf figure 8 ). 

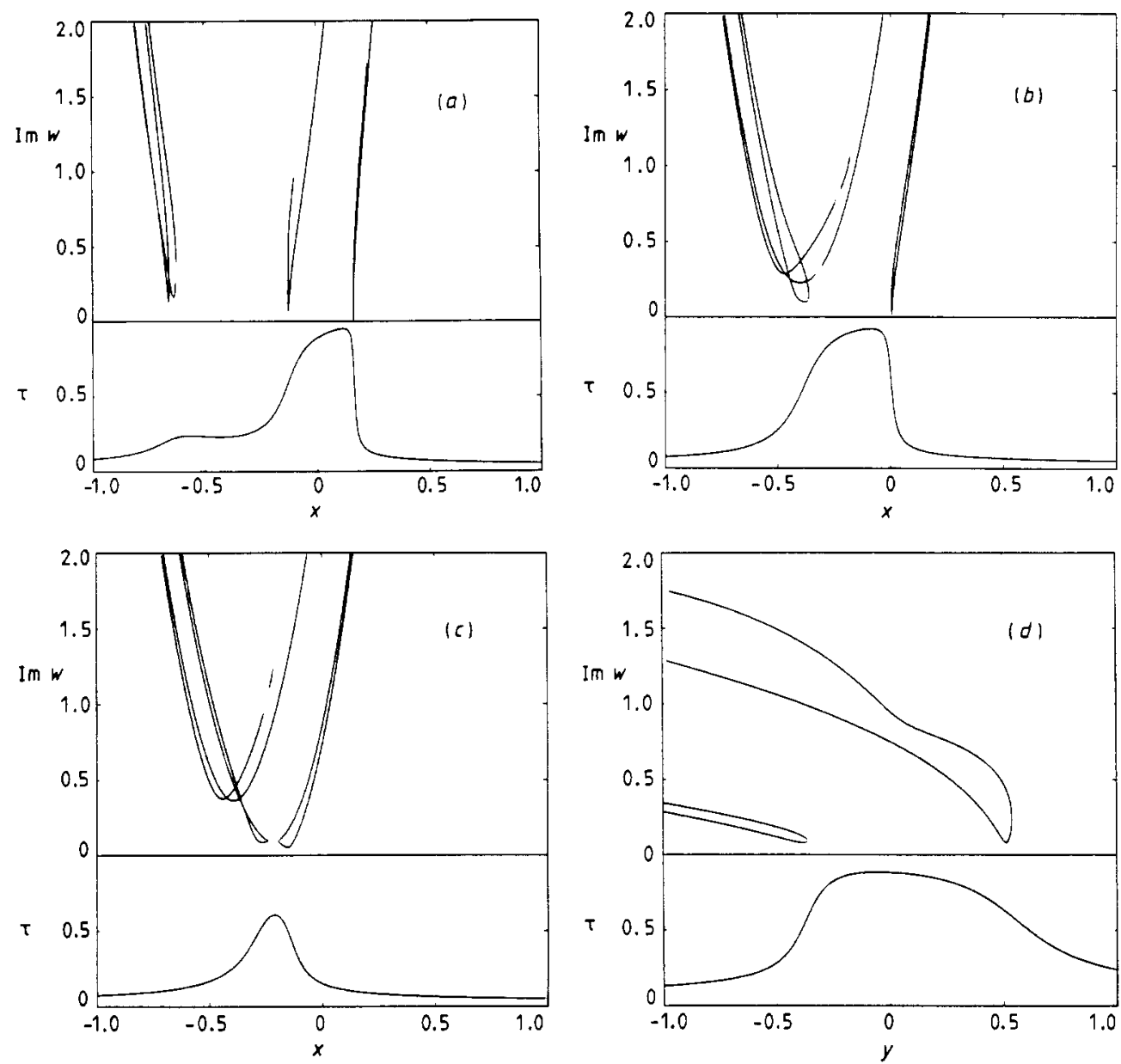

Figure 7. Upper curves show $\|\operatorname{Im}(w)\|$ against the $x$ component of $\operatorname{Re}(w)$ for $(a) y=0.75$, (b) $y=0$, (c) $y=-0.36$, and against the $y$ component of $\operatorname{Re}(w)$ for $(d) x=-0.2$, all for $\beta=10^{-3}$. Lower curves show the trapping time $\tau$ for initial points on the curve $l$.

intersection lines $l$ for figures 6 and 7 are shown in figure 8 , where the manifold $W_{0}^{\mathrm{s}}$ at $b=0.3$ is also shown for comparison.

\section{Final remark}

We have studied transient periodic behaviour in the basin of an attractor at infinity for a quadratic map, which has a saddle-node bifurcation. We are able to predict analytically the region of the initial conditions for which orbits seem to converge to a periodic orbit for a long time, and the duration of these transients.

We conclude that every saddle-node bifurcation, taking place in the basin of a certain attractor, is preceded by transient periodic orbits in this basin. Since saddle-node bifurcations typically occur for dissipative dynamical systems, at least in a range of parameter values (Guckenheimer and Holmes 1983), these transients are a common phenomenon. 


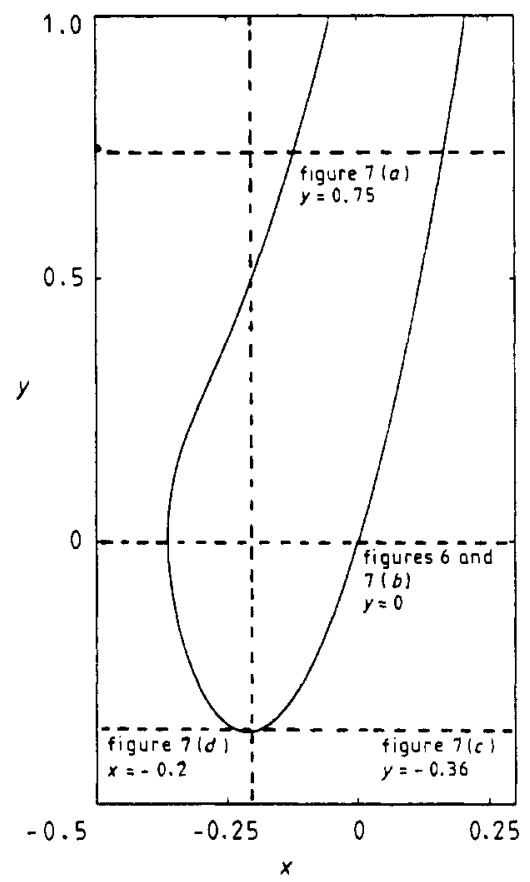

Figure 8. The intersection lines $l$ for figures 6 and 7 . For comparison the manifold $W_{0}^{\text {s }}$ at $b=0.3$ is shown.

\section{Acknowledgment}

One of us (RvD) would like to thank the Stichting Fundamenteel Onderzoek der Materie (FOM) which is financially supported by the Stichting voor Zuiver Wetenschappelijk Onderzoek (ZWO).

\section{Appendix. Construction of invariant manifolds for a quadratic map in $\mathbf{C}^{2}$}

Consider the quadratic map (1) of $\mathbb{C}^{2}$ onto $\mathbb{C}^{2}$ expanded about a fixed point. The map and its inverse can be written, denoting the pair $\{x, y\}$ by $z$, as

$$
z^{\prime}=H z=P z+R z z \quad H^{-1} z=P^{-1} z+Q z z
$$

where $R z z$ and $Q z z$ denote homogeneous quadratic terms.

$P^{-1}$ has eigenvalues $\lambda_{s}^{-1}$ and $\lambda_{l}^{-1}=b^{-1} \lambda_{s}$ (where $\left|\lambda_{s}\right| \leqslant\left|\lambda_{l}\right|$ and $\left|\lambda_{s}\right|<1$ ), and eigenvectors $e_{s}$ and $e_{l}$, respectively.

We prove the following.

Lemma. There exists an entire function $z(u), \mathbb{C}^{1} \rightarrow \mathbb{C}^{2}$, such that

$$
z(u)=H^{-1} z\left(\lambda_{s} u\right), \quad z(u)=u e_{s}+\text { нот. }
$$

Proof. Suppose $z(u)$ to be differentiable: by differentiating (A2) $n$ times and substituting $u=0$ we obtain the hierarchy:

$$
\lambda_{s}^{-n} z^{(n)}(0)=P^{-1} z^{(n)}(0)+\sum_{j=1}^{n-1}\left(\begin{array}{l}
n \\
j
\end{array}\right) Q z^{(j)}(0) z^{(n-j)}(0)
$$


(a superscript $j$ on $z$ denotes $j$ differentiations of $z$ with respect to $u$ ). Here the sum runs from 1 to $n-1$, as $z^{(0)}(0)$ equals zero.

Equation (A3) can be solved explicitly. For $n=1$

$$
\left(\lambda_{s}^{-1}-P^{-1}\right) z^{(1)}(0)=0
$$

which is valid if $z^{(1)}(0)$ points along $e_{s}$. For arbitrary $n>1$ (A3) becomes

$$
\left(\lambda_{s}^{-n}-P^{-1}\right) r_{n}=\sum_{j=1}^{n-1} Q r_{j} r_{n-j} \quad r_{n} \equiv z^{(n)}(0) / n !
$$

Since $n>1$ and $\lambda_{s}$ is the smallest eigenvalue, $\left(\lambda_{s}^{-n}-P^{-1}\right)$ is invertible. Then (A5) immediately yields

$$
\left\|r_{n}\right\| \leqslant q(n-1)\left\|\left(\lambda_{s}^{-n}-P^{-1}\right)^{-1}\right\|_{o p} \max _{j}\left(\left\|r_{j}\right\|\left\|r_{n-j}\right\|\right) \quad n>1
$$

where $q$ is a constant depending on $Q$ and \|\| denotes the norm

$$
\|z\|^{2}=\left(z, S^{+} S z\right)
$$

where $S$ is such that $S P S^{-1}$ is diagonal and (, ) is the usual inner product; \|\|$_{o p}$ is the corresponding operator norm. Then it holds

$$
\begin{aligned}
\left\|\left(\lambda_{s}^{-n}-P^{-1}\right)^{-1}\right\|_{\mathrm{op}} & =\max \left\{\left|\lambda_{s}^{-n}-\lambda_{s}^{-1}\right|^{-1},\left|\lambda_{s}^{-n}-\lambda_{l}^{-1}\right|^{-1}\right\} \\
& \leqslant\left(\left|\lambda_{s}\right|^{-n}-\left|\lambda_{s}\right|^{-1}\right)^{-1}
\end{aligned}
$$

since $\left|\lambda_{s}\right| \leqslant\left|\lambda_{l}\right|$ and $\left|\lambda_{s}\right|<1$.

If we normalise such that $\left\|r_{1}\right\|=q^{-1}$, then we can prove by induction, from (A6) and (A8), that

$$
\left\|r_{n}\right\| \leqslant q^{-1} A^{1-n} \quad A \equiv\left|\lambda_{s}\right|^{-2}-\left|\lambda_{s}\right|^{-1} .
$$

We now can reverse the argument.

Define a function, with $r_{n}$ computed from (A5)

$$
z(u)=\sum_{n=1}^{\infty} r_{n} u^{n}
$$

Obviously $z(u)$ satisfies (A2) on a disc $|u|<A$. However, since

$$
z\left(\lambda_{s}^{-1}(u)=H^{-1} z(u)\right.
$$

the domain of $z(u)$ can be extended to $C^{1}$. Hence $z(u)$ is an entire function (cf also Lauwerier 1984).

For the Henon map (1), write $z(u)=\{g(u), f(u)\}$. From $(1 b)$ one obtains $g(u)=$ $f(\lambda u)$, and thus

$$
z(u)=\left\{f\left(\lambda_{s} u\right), f(u)\right\}
$$

and we compute the Taylor coefficients of $f(u)$

$$
\begin{aligned}
& f(u)=\sum_{n=1}^{\infty} t_{n} u^{n} \\
& t_{1}=1 \\
& t_{n}=\left(\lambda_{s}^{n}+b \lambda_{s}^{-n}-\lambda_{s}-b \lambda_{s}^{-1}\right)^{-1} \sum_{j=1}^{n-1} 2 t_{j} t_{n-j} .
\end{aligned}
$$



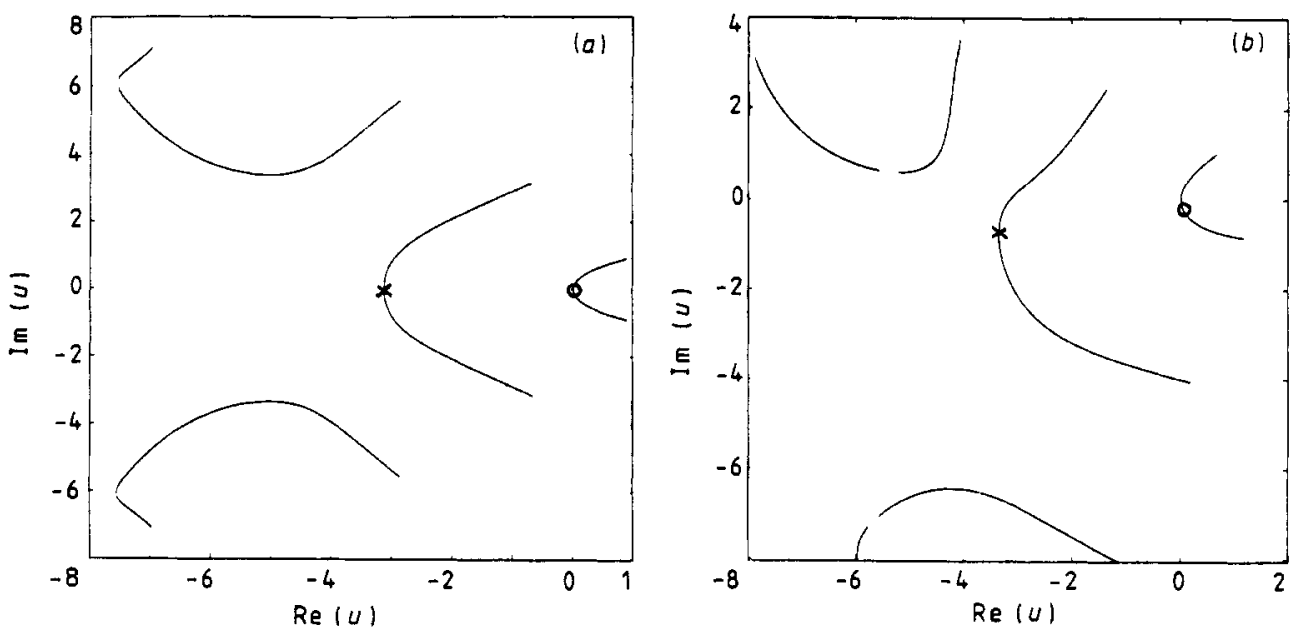

Figure 9. The complex $u$ values which are a solution of (A16), with $y_{c}=0, b=0.3$ for $(a)$ $\beta=0$, (b) $\beta=10^{-3}$. The curves marked with $\times$ and $\bigcirc$ correspond to the branches of $\|\operatorname{Im}(w)\|$ in figures 6 and $7(b)$ which are closest to the real plane.

We used this Taylor expansion for the numerical construction of the complex manifolds; we truncated the expansion at a certain degree, for which the numerical values of the remaining terms were of the order of magnitude of the precision of the computer. Then we solved for $u$ the equation:

$$
\operatorname{Re}(f(u))=y_{c} \text {. }
$$

In this way we obtained solution curves in the $u$ plane (cf figure 9). We constrained ourselves to a relevant range of $x(y)$ values $(|x|<1,|y|<1)$. The projection on $\mathbb{R}^{2}$ obeys $\operatorname{Re}(z)=\left\{x, y_{c}\right\}$ and by construction $z(u) \in \tilde{W}^{s}$ (and analogous for figure $7(d)$, where the $x$ component is kept fixed).

In order to check the validity of the results we varied the truncation order of the Taylor expansion. The solutions in the chosen $x-y$ domain of (A16) were insensitive to such variations.

\section{References}

Amold V I 1977 Geometrical Methods in the Theory of Ordinary Differential Equations (Grundlehren der mathematischen Wissenschaften 250) (Berlin: Springer)

Guckenheimer J and Holmes P 1983 Nonlinear Oscillations, Dynamical Systemms and Bifurcations of Vector Fields (App. Math. Sciences 42) (Berlin: Springer)

Lauwerier H A 1984 Entire functions for the logistic map I Internal report, Department of Applied Mathematics, Mathematical Centre, Amsterdam

Schuster H G 1984 Deterministic Chaos (Mostbach: Physik-Verlag) 